\title{
Comment évaluer un projet de recherche
}

Petite histoire illustrée de l'évaluation de la recherche industrielle à

Pechiney Gp en 1969

Muriel Le Roux

\section{OpenEdition}

Journals

Édition électronique

URL : https://journals.openedition.org/histoire-cnrs/581

DOI : 10.4000/histoire-cnrs.581

ISSN : 1955-2408

Éditeur

CNRS Éditions

\section{Édition imprimée}

Date de publication : 5 novembre 2003

ISBN : 978-2-271-06144-7

ISSN : 1298-9800

\section{Référence électronique}

Muriel Le Roux, "Comment évaluer un projet de recherche », La revue pour I'histoire du CNRS [En ligne], 9 | 2003, mis en ligne le 31 octobre 2006, consulté le 20 mai 2021. URL : http://

journals.openedition.org/histoire-cnrs/581; DOI : https://doi.org/10.4000/histoire-cnrs.581

Ce document a été généré automatiquement le 20 mai 2021.

Comité pour l'histoire du CNRS 


\section{Comment évaluer un projet de recherche $^{1}$}

Petite histoire illustrée de l'évaluation de la recherche industrielle à

Pechiney Gp en 1969

\section{Muriel Le Roux}

1 «Qui vit sans folie n'est pas si sage qu'il le croit ». La Rochefoucauld

2 En 1969, ce qui est devenu Pechiney Gp est le fruit de fusions et d'absorptions. Le secteur chimique d'abord confié à la filiale Pechiney-Saint-Gobain en 1959 vient d'être cédé à Rhône Poulenc. Les entreprises françaises les plus importantes chargées de la transformation de l'aluminium ont toutes été absorbées entre 1964 et 1967. Pechiney $\mathrm{Gp}$, dont la production majeure est l'aluminium primaire, est une firme intégrée depuis la mine de bauxite jusqu'aux produits semi-finis. Le développement international se poursuit en Afrique, aux États-Unis et en Europe. Pour trois ans, jusqu'à la constitution de Pechiney Ugine Kuhlmann en 1971, Pechiney, présent dans le secteur de l'aluminium et de la fabrication des matériaux pour l'industrie nucléaire, est un groupe prospère.

Consolider les parts de marché dans le secteur de la transformation est le maître mot des dirigeants. Si l'expertise scientifique et technique dans le secteur de la fabrication de l'aluminium primaire assure à Pechiney une suprématie technologique mondiale, devançant sa principale concurrente : l'Aluminium Company of America (Alcoa), il n'en va pas de même dans le secteur aval de la fabrication des alliages et de leurs transformations. Aussi, Pierre Jouven, président-directeur général, après avoir achevé l'intégration du secteur de la transformation au groupe, va à nouveau porter son attention sur la recherche. En 1962, il avait envoyé une lettre ${ }^{2}$ à François Ortoli, inspecteur des finances, responsable des questions de recherche auprès du Premier ministre, lui rappelant que la loi-cadre de 1961 créant les actions concertées de recherche et le fonds de développement de 320 millions de francs étaient tout à fait insuffisants pour permettre aux industriels de soutenir l'effort de recherche demandé par le gouvernement, la DGRST et souhaité par l'OCDE.

4 Ayant beaucoup voyagé pour effectuer ce que la gestion des organisations nomme du bench marking, c'est-à-dire des comparaisons entre les différentes modalités 
d'organisation de la recherche industrielle aux États-Unis, il fut l'artisan d'une importante réforme concernant la recherche du groupe.

5 « Un défaut qui empêche les hommes d'agir, c'est de ne pas sentir de quoi ils sont capables ». Bossuet

6 Pierre Jouven créa des structures nouvelles de recherche, pour le secteur de la transformation de l'aluminium. Recrutant un nouveau directeur scientifique (Charles Crussard, polytechnicien du corps des Mines, directeur de recherche à l'Irsid ${ }^{3}$ ) à la fin de l'année 1962, Pierre Jouven avait décidé de créer un grand centre de recherche pour la transformation à Voreppe, près de Grenoble, inauguré en 1966.

7 Le groupe était en pleine recomposition, d'anciens concurrents étaient contraints à travailler ensemble ; ce climat difficile affectait aussi le travail des chercheurs. Afin de faire passer ses idées en douceur, Pierre Jouven fit appel, en 1967, au cabinet américain de consultants Mac Kinsey, pour réaliser un audit général du groupe. La recherche fut l'objet d'un audit spécifique.

8 Les recommandations émises afin que le groupe atteigne la dimension recherchée dans le secteur de la transformation aboutirent à la création d'un département « vente et transformation de l'aluminium » confié à Olivier Bes de Berc (X-Mines). Ce département fut lui-même doté d'une division des recherches de transformation placée sur le même plan que la division marketing.

9 Grâce à l'organisation de cette structure de recherche, Pechiney Gp espère pouvoir rivaliser avec les firmes concurrentes nord-américaines - Alcoa, Kaiser, Reynolds - et augmenter ses parts de marché dans le secteur de la transformation de l'aluminium. D'ailleurs, les dépenses de recherche sont passées de 39 millions de francs en 1966 à 60 en 1969 et 80 en 1970. Les dépenses de recherche engagées étaient couvertes environ à $40 \%$ par les recettes nettes des ventes de brevets, alors que les redevances payées par le groupe étaient de 0,15 million de francs en 1970. Au cours de cette période, l'augmentation de la demande d'aluminium était de 9 à $10 \%$ par an.

10 Outre les enjeux liés directement à la vente de métal, la capacité de répondre à la demande des clients - aussi bien en aviation, en aéronautique, qu'en automobile devait déterminer le futur de l'entreprise. Or, dans ce domaine, les positions de Pechiney $\mathrm{Gp}$ ne sont pas très bonnes et la perspective de perdre des marchés importants se conjugue à celle de devoir acheter des technologies et des licences pour fabriquer des alliages d'aluminium. Ce qui est tout bonnement inconcevable pour la direction générale de l'époque. Aussi la recherche pour la transformation est-elle placée au cœur de la stratégie de la firme.

11 C'est dans ce contexte général qu'il faut replacer le document commenté ici $^{4}$. Il reprend une grande partie des recommandations du rapport rendu par le cabinet Mc Kinsey mais il a été rédigé en collaboration avec la Cegos, cabinet français de consultants et de formation ${ }^{5}$.

12 Le livret classé confidentiel fut utilisé tel que jusqu'en 1973, puis périodiquement remis au goût du jour jusqu'à la fin de la décennie. Il fut diffusé uniquement dans le département de la transformation du métal et de ses alliages de Pechiney.

13 L'ensemble représente un savant dosage entre la culture française et la culture américaine de gestion de la recherche. Le ton se veut détendu afin de mettre l'évaluateur dans une position d'objectivité destinée à servir les desseins de l'entreprise. Des citations de La Rochefoucauld, Graham Greene, Bossuet, Fénelon, 
Oscar Wilde, Albert Einstein, Vauvenargues, servent de ligne directrice. Elle est relayée par des aphorismes qui légendent les dessins humoristiques chargés de mettre en confiance l'évaluateur sur lequel repose le principe de sélection des projets de recherche et donc à moyen terme la capacité innovatrice de la firme.

Il est intéressant de noter que les illustrations elles-mêmes définissent les spécificités du principe d'évaluation de la recherche industrielle.

En introduction est affirmé le but de l'évaluation qui est de transmettre le plus vite possible au service marketing ou opérationnel l'idée qui vient de germer. À aucun moment n'est défini ce que devrait être la recherche. Le postulat de départ était qu'il ne s'agissait que de recherche appliquée, même si dans les faits, la recherche fondamentale était loin d'être absente. Ainsi, l'évaluateur n'est pas un chercheur, ni même un responsable de programme, c'est « un opérationnel ».

Il s'agissait souvent d'un cadre de la branche marketing, connaissant bien la demande, les besoins des clients et le marché. Il devait être capable de faire remonter la demande vers les laboratoires de recherche.

La fiche d'évaluation doit permettre de situer l'idée d'innovation sur le marché futur. Aussi le premier chapitre du livret est-il consacré au marché, aux objectifs commerciaux de la recherche.

Viennent ensuite le chapitre sur la recherche elle-même, puis celui traitant de la rentabilité. Les aspects scientifiques sont évoqués (traitant des relations avec la recherche publique).

Enfin, le livret se conclut sur la détermination d'un indice de priorité qui permet de classer les programmes de recherche entre eux.

Dans une entreprise où la culture dominante était celle des ingénieurs de l'électrolyse qui avaient réussi à garder la recherche dans leur giron jusqu'à la fin des années 1960, les procédures conduisant à individualiser la recherche pour la confier à un personnel spécifique en des lieux autonomes furent très mal vécues. Aussi fallut-il organiser régulièrement des journées consacrées à l'évaluation de la recherche. Il fallait contrôler sans les brider à l'excès les chercheurs qui très vite ont su mettre en place des stratégies pour s'approprier l'organisation, les programmes et les budgets afin de travailler sur des thèmes qui les intéressaient en dehors des besoins des services opérationnels.

21 Rationaliser l'activité des centres de recherche par un texte servant d'outil de référence afin d'empêcher une dérive des chercheurs tout en restituant le rôle de la décision finale qui revenait aux ingénieurs, tels étaient les buts de cette plaquette.

Car chez Pechiney, dans les années 1960 et 1970, il n'y a plus de place pour la recherche exploratoire sur le long terme. L'entreprise a complètement intégré les nouvelles techniques de management. Nous sommes dans un système business où la recherche doit être drivée par le market-push et dans lequel il faut lutter contre le research-pull. Tels sont les maîtres mots des directives émises par la direction de la recherche de transformation dirigée par des partisans des méthodes américaines de management et adepte du franglais. Évaluer non pour mettre en valeur des connaissances, mais pour répondre à la demande du marché, voire anticiper sur les pressions qu'il exerce, telles sont les missions de l'évaluateur. Dans ce contexte, il est clair que l'on n'évalue que les projets futurs... 

la fois des principes théoriques et des savoir-faire techniques, en favorisant une collaboration avec les services opérationnels (marketing et industriel). Se dégage ainsi un principe organisateur qui tend à systématiser l'investigation et permet d'en programmer le déroulement grâce à des méthodes inspirées des recherches opérationnelles de développement.

Cette notice fut l'une des premières en France à " formaliser " les principes de l'évaluation de la recherche industrielle. Auparavant, les supports étaient américains. On peut sans doute y voir l'influence de la Cegos. Elle fut reprise par d'autres groupes industriels. Elle montre tout l'intérêt porté aux moyens recherchés par les dirigeants pour anticiper et gérer l'innovation. D'ailleurs, l'année suivante, Pechiney accueillait une équipe de chercheurs (Michel Bauer, Michel Callon, Élie Cohen, Lucien Karpik et alii) du Centre de sociologie de l'innovation de l'École des Mines de Paris, qui, en collaboration avec le CNRS et le Cordes, organisme dépendant du ministère de l'Industrie, allait mener de nombreuses enquêtes afin d'analyser les stratégies des producteurs d'aluminium dans le monde. Les conclusions de l'un des principaux rapports étaient qu'il fallait pouvoir garder un équilibre entre science et technique, entre recherche théorique et exploratoire et recherche business. Malgré ces efforts et le constat de quelques réussites, le système ne fut pas pleinement performant. Au moment où le groupe européen de l'aéronautique se constituait, il devenait déterminant que Pechiney puisse être présent sur tous les marchés de la transformation des alliages d'aluminium. Si l'évaluation était l'une des conditions d'une recherche performante, elle n'était pas la seule. Il fallait que la R\&D soit reconnue comme une activité stratégique par l'ensemble des dirigeants de la firme. Les assises de la recherche et de la technologie provoquées par le ministre Jean-Pierre Chevènement, auxquelles participèrent certains responsables de la recherche du groupe, modifièrent le climat général en France. Les industriels français reconsidérèrent la place de la R\&D au sein de leurs firmes.

L'embauche, en 1984, d'un directeur de recherche du CNRS comme directeur de la R\&D devait permettre de réfléchir à la mise en place de nouveaux équilibres en la matière tant il est vrai que Pechiney avait perdu une grande partie de son assise scientifique dans la pratique de ses métiers.

26 S'il était un point sur lequel dirigeants et consultants étaient d'accord, c'était bien celui de la difficulté de l'évaluation de la recherche. Toute la rationalité, toutes les fiches d'évaluation, toutes les modélisations, toutes les prévisions n'empêcheront pas que l'innovation émerge ailleurs ainsi que la citation d'Einstein le rappelait : « L'imagination est plus importante que la connaissance ».

27 L'art de manager, de diriger la recherche industrielle consiste justement à trouver le compromis entre investissements et rentabilité, entre liberté créatrice et soumission à la production, l'évaluation ne devant pas étouffer l'idée dans l'œuf...

L'histoire de la R\&D industrielle a prouvé le bien-fondé de la pratique de " l'autocritique constructive » qui fut instaurée à partir de 1984.

Notons, toutefois, qu'en matière d'évaluation de la R\&D, la mémoire et la transmission des pratiques connaissent des solutions de continuité dans le temps. Contrairement à ce que l'on observe lorsqu'il s'agit de conflits sociaux ou de problèmes environnementaux, tout se passe comme si le management de la recherche n'était pas

La revue pour l'histoire du CNRS, 9 | 2003 
transmissible. En effet, au début des années 1990, le directeur de la R\&D engagea une réflexion sur la définition des buts stratégiques de la recherche. L'évaluation des projets et des résultats constitua une large part du rapport. L'argumentaire, les consignes et les directions n'étaient pas différents de ceux de la brochure présentée ici. Seuls les dessins et les aphorismes avaient disparu. J'ai interrogé les responsables de la R\&D des années 1990 afin de savoir pourquoi ils n'avaient pas utilisé cette brochure. L'un m'a répondu que « tout cela était dépassé », le second m'a dit qu'il se souvenait de cette brochure mais qu'il n'avait pas songé à l'utiliser. Enfin le dernier, après l'avoir lu, dit que s'il en avait connu l'existence, il aurait sans doute utilisé ce document comme base de travail d'un séminaire.

Si cette brochure devait fournir aux cadres responsables de la R\&D des éléments leurs permettant de motiver les chercheurs, si ses principes sont toujours d'actualité, il est certain qu'aujourd'hui, le ton et certains dessins ne seraient pas publiés en l'état surtout lorsqu'il s'agit d'utiliser l'image des Africains ou celle des femmes...

31 «Combien d'occasions, d'éclairs de lucidité l'on perd, simplement parce qu'une besogne n'est plus qu'une besogne ». Graham Greene

\section{NOTES}

1.Le document dont nous présentons des extraits est un livret de vingt-huit pages daté de juin 1969.

2.Lettre publiée dans M. Le Roux, Un siècle de recherche industrielle à Pechiney, Paris, Éditions Rive droite, 1998, 499 p., p. 307-308.

3.Institut de recherche de la sidérurgie, inauguré en 1950.

4.Illustré par un membre du personnel de Pechiney Gp.

5.A. Weextsten, « Histoire de la Cegos », doctorat de l'EHESS sous la dir. de P. Fridenson, Paris, 1998.

\section{INDEX}

Mots-clés : CNRS, évaluation, recherche, projet, recherche industrielle, Péchiney Gp 


\section{AUTEUR}

\section{MURIEL LE ROUX}

Muriel Le Roux est chargée de recherche au CNRS à l'Institut d'histoire moderne et contemporaine (CNRS-ENS). Elle vient de publier « Genèse des textes de Pierre Potier, chimiste des substances naturelles » Genesis, ${ }^{\circ}$ 20, 2003, p. 91-125 et avec Odile Welfelé, "Les archives scientifiques contemporaines et l'écriture de la science. Le cas du CNRS ", ibid., p. 167-177. Elle prépare actuellement un ouvrage sur l'ICSN. 